AROUEOLOGía Y SOCIEDAD

№ 28,2014 : $335-354$

ISSN: 0254-8062

RECIBIDO: MARZO DE 2014

ACEPTADO: SETIEMBRE DE 2014

\title{
LA BIOLOGÍA EN LA TOPONIMIA DE PALLASCA
}

\author{
MARÍA del CARMEN CUBA M. \\ UniVERSIDAD NACIONAL MAYOR DE SAN MARCOS \\ mercycubam@yahoo.com
}

\section{RESUMEN}

Las especies biológicas se desarrollan en pisos ecológicos determinados. Las personas, al comunicarse procesan cognitivamente la relación biología-medio geográfico, y la fijan en su lengua a través de nombres propios o topónimos. El propósito del presente estudio es mostrar que estos topónimos, por un lado, reflejan las entidades biológicas acordes a los pisos ecológicos y, por otro, constituyen un testimonio de lenguas y prácticas de pueblos desaparecidos en la provincia de Pallasca (Áncash).

Los datos son parte de un corpus vasto recopilado in situ, en el 2012; obtenido de varios informantes de diferente nivel social y grado de estudios; básicamente mayores. Luego estos fueron seleccionados y clasificados según fuera su referente una entidad biológica: animal o planta y según su ubicación en alguna de las «cuatro» regiones naturales que posee la zona de estudio.

Los resultados muestran un total de 136 topónimos, de los cuales $100(73,53 \%)$ aluden a nombres de plantas y 36 (26,47\%), a aquellos de animales. La región que contiene mayor cantidad de topónimos con referencia a entidades biológicas; e. i, con nombres de plantas o de animales, es la región quechua: 44 (32,35\%) de plantas y 13 (9,56\%), de animales. Le sigue la suni o jalca: $26(19,12 \%)$ de plantas y $26(19,12 \%)$, de animales. Después la yunga: $29(21,32 \%)$ de plantas y el $1(0,74 \%)$, de animales. Por último, la región ruparupa: 8 $(5,88 \%)$ de plantas solamente. En cuanto a su etimología: 35 (25,74\%) nombres son de origen castellano, 65 (47,79\%), quechua; 11 (8,09\%), culle y 25 (18,38\%), de origen incierto.

Palabras Clave: Toponimia, regiones geográficas, biología, lingüística, Pallasca

\section{ABSTRACT}

The biological species develop in determinate ecological levels. The people, when communicating, process cognitively the relation biology and geography, and fix it in his tongue through proper names or toponyms. The purpose of the present study is to show that these toponyms, on the one hand, they reflect the biological entities that accord to the ecological levels and, by another, constitute a testimony of tongues and practics of missing villages in the province of Pallasca (Áncash).

The data are part of a vast corpus collected in situ, in the 2012; obtained from several informants of different social level and degree of studies; basically elder. Then these were selected and classified according to a biological entity: animal or plant and according to their location in any of the «four» natural regions of the area of study.

The results show a total of 136 toponyms, of which 100 (73,53\%) contain names of plants and 36 (26, $47 \%)$, of animals. The region that contains greater quantity of toponyms with reference to biological entities is the quechua region: with $44(32,35 \%)$ names of plants and 13 (9,56\%), of animals. Followed by the suni 
or jalca: $26(19,12 \%)$ names of plants and $26(19,12 \%)$, of animals. Afterwards the yunga, with $29(21,32 \%)$ names of plants and the $1(0,74 \%)$, of animal. Finally, the ruparupa region, with $8(5,88 \%)$ names of plants only. With respect to etymology: $35(25,74 \%)$ names are of Spanish origin, $65(47,79 \%)$ are quechua; 11 $(8,09 \%)$, culle and 25 (18,38\%), of uncertain origin.

KEYworDs: Toponymy, geographic regions, biology, linguistic, Pallasca.

\section{INTRODUCCIÓN}

El presente artículo presenta una muestra toponímica de la provincia de Pallasca, cuya referencia alude tanto a elementos fitonímicos como a zoonímicos, propios de la zona. Lo que trata de mostrar este estudio es que los nombres propios de lugar que se han humanizado en esta región, armonizan con los pisos ecológicos de la sierra andina. La clasificación del espacio geográfico de la zona de estudio tiene como base "Las ocho regiones naturales" que ofrece Javier Pulgar Vidal (2007), cuya adaptación da como resultado cuatro regiones o pisos ecológicos. Estas cuatro regiones son: 1) yunga fluvial; 2) quechua; 3) suni o jalca (que incluye parte de puna); y 4) ruparupa o Selva Alta. El corpus total, es decir, los fitotopónimos y zootopónimos están distribuidos dentro de las cuatro regiones propuestas o adapatadas. Los topónimos irán ordenados formando pequeños grupos de relación de palabras y en orden alfabético. Al final se hará un estimado sobre las lenguas que conforman esta muestra de topónimos.

La superficie terrestre, muchas veces anónima, se humaniza e ingresa en el discurso social a través de los topónimos, los que, por un lado, constituyen una forma de apropiación del espacio geográfico con sus recursos propios, y por otro, una reivindicación cultural y social de los parajes, montañas, cuevas, lagunas, ríos, etc. En los topónimos se da el encuentro de espacio y tiempo mediados por la memoria llamada memoria histórica (Pinchemel 1979).

En lo lingüístico y cultural, el paisaje ecológico pallasquino revela lo autóctono y hereditario: culle y quechua (Chugurmaca, Tauribara, Pichungoñ, Condormuchca) y también lo impuesto y sobrevenido: hispano (Sauce, Cortadera, Pajarito, Perdiz).

\section{BREVE DESCRIPCIÓN DE LA ZONA DE ESTUDIO}

Pallasca es una provincia ubicada al norte de Ancash, entre los paralelos $8^{\circ}$ y $9^{\circ}$ (latitud sur) y los meridianos $77^{\circ}$ y $78^{\circ}$ (longitud oeste) (INGP). La altura oscila entre los 1000 y $4100 \mathrm{msnm}$, delimitada por el norte, por el río Marañón, por el oeste por el río Tablachaca, que confluye en el Chuquicara y por el este, por el río Santa. Limita por el suroeste con la provincia de El Santa; por el sur, con Corongo y; por el sureste, con Sihuas.

Políticamente la provincia pertenece al departamento de Ancash y se ubica en su extremo norte. Cuenta con once distritos: Bolognesi, Cabana, Conchucos, Huacaschuque, Huandoval, Lacabamba, Llapo, Pallasca, Pampas, Santa Rosa y Tauca.

A excepción de Huacaschuque y Llapo, todos los distritos se distribuyen en suelos de variada ecología, sin llegar ninguno a alcanzar las tradicionales categorías de región «costa» ni «selva baja» ni tampoco «janca o cordillera».

Históricamente, en épocas preincaicas, esta zona, correspondiente a los Conchucos, compartía con la sierra de los actuales departamentos de La Libertad y de Cajamarca, por haber sido un amplio territorio que acunó a la cultura y lengua culle. Pallasca ha sido el último bastión de la sociedad culle. La lengua y cultura de esta sociedad se encontraban en plena vigencia en el siglo XVII cuando arribaron los españoles con una lengua y cultura mucho más extrañas que las llevadas por la sociedad conquistadora incaica, hablante del quechua. Se supone que la sociedad de los Conchucos estaba entrando a una etapa de bilingüismo culle-quechua, pero con la penetración abrupta de los 
hispanos y la extirpación de idolatrías a través de la religión católica se produjo primero una suerte de transculturación del culle, quechua y castellano, y finalmente se impuso la sociedad hispana con el castellano. Sin embargo, a pesar de que la cultura hispana, a través de la iglesia católica, ha tratado de borrar drásticamente las prácticas culturales y lingüísticas de las sociedades culle y la quechua, estas culturas nativas dejan entrever vivamente sus huellas en muchas esferas de la vida cotidiana, y especialmente en la toponimia.

\section{LAS REGIONES O PISOS ECOLÓGICOS Y LA BIOLOGÍA EN PALLASCA}

La provincia de Pallasca presenta un suelo de relieve muy accidentado y un clima variado, ofreciendo una flora y fauna amplias, excluyendo lo que correspondería a las tradicionales regiones de la costa y de la selva baja.

Con el propósito de identificar los topónimos que hacen referencia a entidades biológicas según su hábitat, se tendrá como base la clasificación geográfica que hace Javier Pulgar Vidal, quien considera para el Perú ocho regiones naturales o pisos ecológicos, a saber: 1) Chala (costa), 2) yunga, 3) quechua, 4) suni, 5) Puna, 6) janca o cordillera, 7) ruparupa o selva alta y 8) omagua o selva baja.

Esta clasificación será adaptada en razón de la altura y la temperatura; así como del relieve, el suelo y también de la flora y la fauna; de modo que se tendrá solo cuatro regiones, a saber: 1) yunga fluvial, 2) quechua, 3) suni o jalca y parte de puna y 4) ruparupa o selva alta.

1. Yunga fluvial: Desde 1000 hasta $2300 \mathrm{msnm}$. La palabra yunga o yunca, significa «valle cálido». Relieve: valles estrechos y quebradas profundas. Clima templado cálido, con abundantes precipitaciones. El clima de la zona yunga fluvial es menos caluroso que el de la yunga marítima. Abundante flora y variedad de pájaros. Flora: el molle, el cactus, la cabuya, los helechos, las orquídeas. Son productos límite de la yunga la palta, lúcuma, guayaba, chirimoya, naranja, caña de azúcar, etc. Fauna: aves como el chaucato y el taurigaray.

2. Quechua: Desde 2300 hasta $3500 \mathrm{msnm}$. La palabra quechua significa 'valle templado'. Relieve escarpado con valles interandinos. El clima es templado y seco. Flora: el aliso, el eucalipto, la tara, etc. Se cultiva calabaza, arracacha, caihua, maíz, papa, entre otros.Son productos límites el trigo, el manzano y el membrillo. Fauna diversa: el halcón, el gorrión andino, el venado, el puma, etc.

3. Suni o jalca (y parte de puna): Entre 3500 y 4100 msnm. La palabra suni en quechua es 'alto'; jalca viene del quechua sallqa 'silvestre, salvaje, tierra desierta'. Relieve rocoso e inclinado. Clima templado-frío, con precipitaciones sólidas. Se producen las llamadas «heladas». Flora: cola de zorro, cantuta, quinales, taya, saúco. Tiene como productos límite el olluco y la oca. Se cultiva mashua, quinua, maca, cañihua o cañagua, haba, y tarwi. Fauna: Destacan zorros, vizcachas, zorrillos o añases, venados, cuyes, osos, zorzales negros, sapos, gavilanes, hasta el cóndor y el puma. La trucha es característica en las lagunas.

Clima: Desde el templado-frío hasta el frío, con gran sequedad ambiental y precipitaciones estacionales de lluvia y granizo.

En la Puna hay baja presión atmosférica, menor difusión de oxígeno en el aire y clima frígido, con escasas precipitaciones y una temperatura media anual de $6^{\circ} \mathrm{C}$ hasta $7^{\circ} \mathrm{C}$.

4. Ruparupa o Selva Alta: El nombre ruparupa significa 'ardiente' o 'lo que está caliente'. Esta región se encuentra situada al otro lado de los Andes, en su flanco oriental, extendiéndose desde los $400 \mathrm{msnm}$. Su relieve es bastante accidentado destacando la presencia de pendientes, las más bajas de la Cordillera de los Andes, en su flanco oriental.

El clima es cálido-húmedo. Las precipitaciones en esta zona son abundantes entre los meses de diciembre y marzo. La temperatura media anual es de $22^{\circ} \mathrm{C}$ a $25^{\circ} \mathrm{C}$, siendo la máxima de $35^{\circ} \mathrm{C}$. La 
flora de está conformada por una vegetación bastante densa. Las principales especies vegetales del bosque son el cedro, el palo santo, etc. La fauna es muy variada. Son exponentes el venado; reptiles y diferentes aves. En Pallasca, esta región la constituyen las riberas del Marañón, en la frontera con La Libertad. La actividad principal de esta región es la agricultura. Se cultiva café, coca y frutales.

Este estudio se centrará en los elementos biológicos propios de la zona.

\section{Metodología}

Inicialmente la recopilación de datos se comenzó a hacer a partir de las hojas Carta Nacional (escala 1: 100.000) del Instituto Geográfico Nacional del Perú, en el sistema UTM, pero al verificar que habían algunos errores en la forma de los nombres, se optó por hacer primero el trabajo de campo.

Los datos son parte de un corpus vasto recopilado in situ, en el 2012; obtenido de varios informantes de diferente nivel social y grado de estudios; básicamente mayores. Se usó un GPS para determinar los paralelos y meridianos así como la altura de los lugares más relevantes de la provincia. También máquina fotográfica y una grabadora digital cuando algún informante ofrecía algún relato o referencia importante acerca del lugar. El grueso de los datos fueron seleccionados y clasificados según fuera su referente una entidad biológica: animal o planta y según su ubicación en alguna de las «cuatro» regiones naturales que posee la zona de estudio y, de esta submuestra se ha elegido al azar una muestra más pequeña.

\section{LA TOPONIMIA: FUENTE DE CONOCIMIENTO DEL MEDIO AMBIENTE}

Este trabajo tiene como como primer objetivo mostrar la capacidad que tiene el hombre para transformar culturalmente su medio ecológico; particularmente los elementos biológicos asociados a los fenómenos geográficos mediante los topónimos. Un segundo objetivo se centra en los mismos topónimos, cuyo propósito final es ofrecer el desarrollo del sentido de identidad y pertenencia en los territorios, ya que la toponimia es un bien patrimonial, reflejo del medio ambiente local y su historia.

«La toponimia no es solo la historia de los nombres propios más usuales en un idioma, pues encierra, además, un singular interés como documento de las lenguas primitivas, a veces los únicos restos que de algunas de ellas nos quedan. Los nombres de lugar son viva voz de aquellos pueblos desaparecidos, transmitida de generación en generación, de labio en labio, y que por tradición ininterrumpida llega a nuestros oídos en la pronunciación de los que hoy continúan habitando el mismo lugar, adheridos al mismo terruño de sus remotos antepasados.» (Menéndez Pidal 1952: 6).

Los nombres de lugar componen una enciclopedia selectiva y una cartografía mental, de elaboración local, donde se plasma el modo en que los nativos perciben el entorno, se comunican entre ellos acerca de él y extraen utilidades. A través de la toponimia se hace visible qué rasgos del territorio eran o son considerados significativos para la población local.

El valor de la toponimia está en que esta nos revela conocimientos y saberes que van más allá de los límites del dominio puramente lingüístico, para lo cual es necesario la intervención de otras disciplinas que participan de la cultura local tales como la biología, la geografía, la historia, la arqueología, la antropología, etc.

Precisamente este estudio destaca los topónimos asociados a los seres biológicos desarrollados y producidos en los diferentes pisos ecológicos en que se asienta la provincia de Pallasca.

En el corpus analizado se puede verificar de qué modo es organizado como un todo perceptivo el conjunto de plantas y animales del medio; de las oportunidades de cultivar y criar en cada uno de los pisos ecológicos que tienen los pobladores pallasquinos (cf. Afable, P.O. y Beeler, M.S., 1996). 
La continua tarea que agricultores y, en general, campesinos y pastores, durante siglos han venido sucediéndose en este territorio, se plasma en un corpus toponímico que revela "prácticas y conocimientos», en gran parte soslayados por la superposición de saberes foráneos de diferentes épocas, muy extraños para los habitantes de localidades nativas (Pinchemel y Beeler 1979).

En el territorio de estudio se ha encontrado muchos topónimos cuya etimología proviene de entidades fitonímicas y zoonímicas que «informan de prácticas útiles o significativas de sociedades ahora extintas» (Íbidem).

\section{FORMA DE ABORDAR EL INVENTARIO LÉXICO}

Para la presentación del corpus se ha seguido de alguna manera el modelo de Herman Carvajal Lazo, en su trabajo Ovalle y la toponimia indígena del Limarí (1998). Dentro de este marco, cada topónimo, será descrito en el orden que se indica:

1. En negrita, el nombre del topónimo y variantes léxicas (si hubieran).

2. Aparición en el Diccionario de la Real Academia Española, en su última edición (indicada por un asterisco entre paréntesis).

3. Frecuencia del topónimo (indicada por un número entre paréntesis).

4. Tipo de fenómeno geográfico.

5. Ubicación del fenómeno geográfico dentro de la provincia (indicada por la sigla del distrito entre paréntesis).

6. Desagregado de la palabra en sus morfemas simples (si se trata de una palabra compuesta o derivada).

7. En negrita, forma registrada o no en los diccionarios u enciclopedias.

8. Etimología y significado ${ }^{1}$.

9. Descripción y otros datos relativos a los elementos biológicos tomados del DRAE, del Diccionario Enciclopédico de Plantas Útiles del Perú (DEPUP) o, en algunos casos, propuestos en forma particular (esto último, cuando los datos no están en estas fuentes mencionadas o si lo están, no se adecúan a los que aparecen en Pallasca).

10. Nombre científico ${ }^{2}$.

El inventario de topónimos será analizado en pequeños grupos de relación de palabras. En un primer caso, tendrá como base el nombre del vegetal o del animal que alude y si el nombre tuviera elementos lingüísticos que lo preceden, estos irán a su derecha, inmediatamente después de una coma. Por ejemplo, los topónimos Molle, El Molle, Loma del Molle, Los Molles, Pampa del Molle, Mollebamba y Mollepampa; aparecerán en el inventario, tal como sigue:

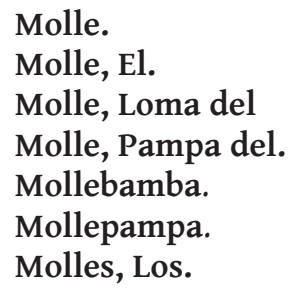

1 La etimología, en la mayoría de casos se han tomado del DRAE (Diccionario de la Real Academia Española), el DQAH (Diccionario Quechua Ancash-Huaylas), el Vocabulario de la lengua general de todo el Perú llamada lengua qquichua o del inca de González Holguín (1989 [1952]), de los trabajos de Torero (1989), Flores (2000), Cuba (2005) y a veces de fuentes virtuales.

2 El nombre científico, en la mayoría de casos se ha tomado del DEPUP, otras veces del libro Fitonimia. 1100 plantas medicinales; raras veces del DRAE o de otras fuentes. 
donde los pasos 8) 9) y 10); es decir, la etimología y significado; la descripción de la planta o del animal y los demás datos relacionados con el conocimiento que el poblador tiene acerca de este; así como el nombre científico, irán acompañando solamente a la primera forma que ocuparía en el orden alfabético; es decir, estos datos acompañarán solamente a Molle. Para las otras entradas solo habrá una remisión (Ver Molle).

Entonces se tendrá:

Molle. (*), (1), chacra, (Pam.), molle. (Del q. mulli). m. Árbol de mediano tamaño, de la familia de las anacardiáceas, propio de América Central y Meridional, que tiene hojas fragantes, coriáceas y muy poco dentadas, flores en espigas axilares, más cortas que las hojas, y frutos rojizos. Su corteza y resina se estiman como tonificantes nerviosos y antiespasmódicos (DRAE). En la zona de estudio se usan las hojas en infusión y en baños para curarse de los bronquios. m. Nombres comunes: árbol de la vida, cullash, falsa pimienta, huigan, huiñan, pimienta del Perú, maera, orcco mulli. NC: Schinus molle.

Molle, El. (2), quebrada y loma, (Huand.). Ver Molle.

Molle, Loma del. (1), loma, (Pam.). Ver Molle.

Molle, Pampa del. (1), chacra, (Pam.). Ver Molle.

Mollebamba. (1), chacra, (T.), molle y bamba. Ver Molle.

Mollepampa. (1), chacra, (Con.), molle y pampa. Ver Molle.

Molles, Los. (4), paraje, quebradas y cerro, (S.R. y Pam.). Ver Molle.

Si, en un segundo caso, se tienen los topónimos Hualango y Hualanguito, la descripción consistirá en lo siguiente:

Hualango. (1), chacra (Con.), huarango. (Del q. waranqu). m. Árbol mediano, copa extendida, espinoso; hojas compuestas finas; flores en cabezuelas amarillas; vainas anchas y encorvadas (DEPUP). NC: Acacia macracantha.

Hualanguito. (1), chacra (Con.); diminutivo de Hualango. Ver Hualango.

En este ejemplo se observa que:

a. En la primera entrada de este par de topónimos hay ausencia de (*); es decir, la forma hualango no está registrada en el DRAE; tampoco está la forma huarango que sí lo registra el DEPUP y que, por lo tanto, este se convierte en fuente de referencia. Entonces para el paso 6) se colocará huarango, a partir del cual se tomará su descripción y nombre científico.

b. En la segunda entrada, Hualanguito es una forma no registrada en los diccionarios, por lo que habría que colocar en la sección que corresponde a 6) diminutivo de Hualango y después una remisión a la entrada anterior: Ver Hualango.

Si, en un tercer caso, se tiene el topónimo como Culantrillo.

Culantrillo. (*), (1), chacra, (Con.), culantrillo. m. Helecho perenne; rizoma escamoso; radículas; peciolos largos, negros, radicales, delgados; hojas bi-tripinnadas, delgadas, verde-amarillentas, lobuladas, en abanico; esporangios en el margen superior de las hojas, color ocre, soros, reniformes (DEPUP). NC: Adiantum capillus-veneris. 
Si, en un cuarto caso, se tienen un topónimo como Shayle:

Shayle, El. (1), chacra (Bol.), shayle. (De or. inc.). m. Planta de $2.5 \mathrm{~m}$ de altura, flores amarillentas o verdes, inflorescencia de $80 \mathrm{~cm}$ de alto, dispuestas en forma de espiga. En su conjunto es una planta pegajosa y de olor no agradable. De uso medicinal. Antiguamente, en la zona, sus hojas han sido utilizadas para reemplazar al tabaco. NC: Nicotiana glutinosa.

se aprecia que la descripción es propia; no se ha tomado de ninguna fuente porque ninguna de las consultadas la registra.

Si, en un quinto caso se tiene Chugán y Chugañ referidos a un mismo lugar, se registrará así:

Chugán Chugañ, El. (1), chacra, (Cab.), chugañ. (Probablemente de origen culle.). m. Arbusto de ramificación espinosa, de consistencia dura, de frutos pequeños de color rojo, de consistencia de la manzana verde. El topónimo se refiere a una chacra rodeada de chuganes. Mayormente esta planta es utilizada para impedir entradas a las chacras con sementera en maduración. NC: Hesperomeles cuneata.

donde se observa que ambas formas son solo variantes fonéticas que deben ir en la entrada unidas por el signo .

\section{INVENTARIO TOPONÍMICO SEGÚN LAS REGIONES} ECOLÓGICAS Y SEGÚN LOS GRUPOS BIOLÓGICOS

\section{ABREVIATURAS}

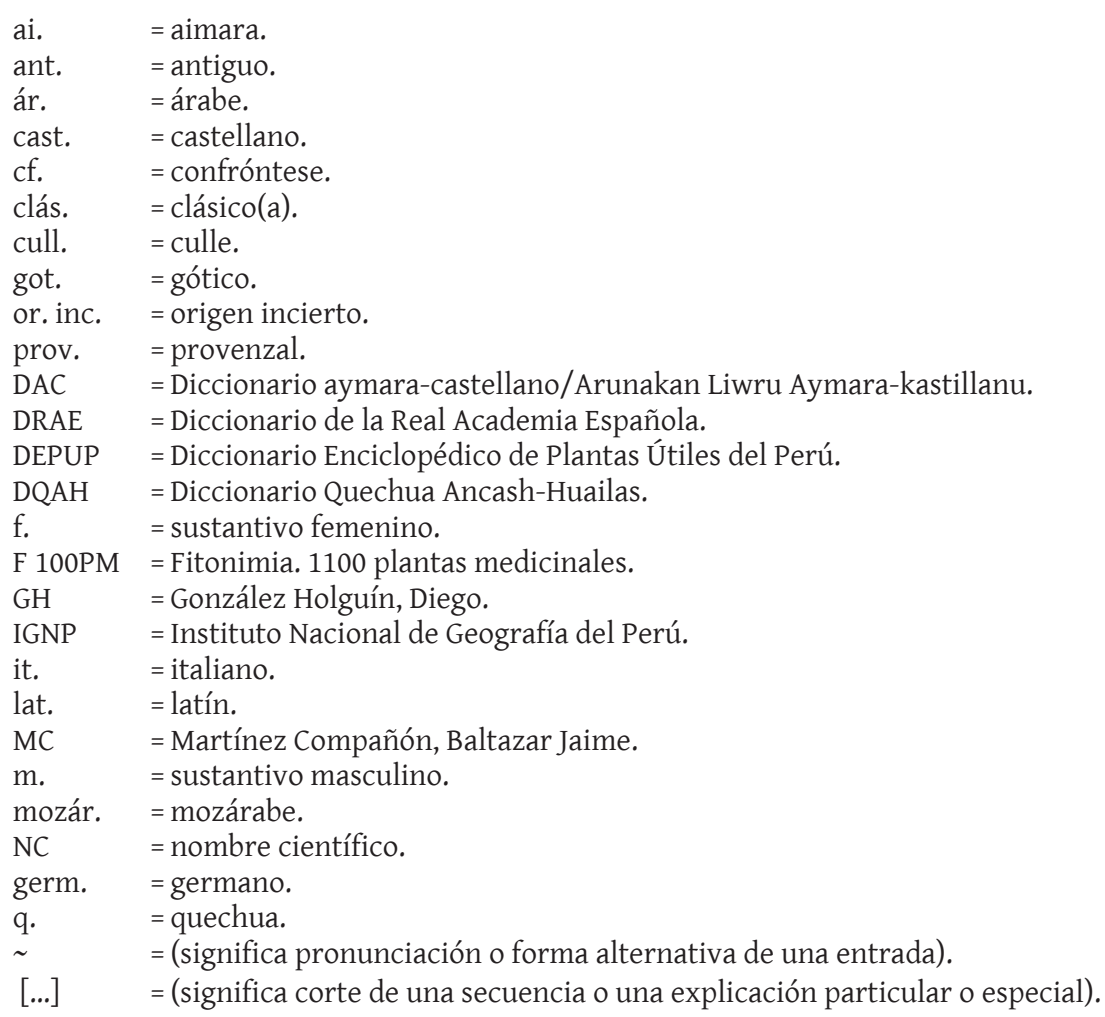


Los distritos de Pallasca son once y tendrán las siguientes abreviaturas:

$\begin{array}{ll}\text { Bol. } & =\text { Bolognesi. } \\ \text { Cab. } & \text { = Cabana. } \\ \text { Con. } & \text { = Conchucos. } \\ \text { Huac. } & \text { = Huacaschuque. } \\ \text { Huand. } & \text { = Huandoval. } \\ \text { Lacab. } & \text { = Lacabamba. } \\ \text { Lla. } & \text { = Llapo. } \\ \text { Pall. } & \text { = Pallasca. } \\ \text { Pam. } & \text { = Pampas. } \\ \text { S.R. } & \text { = Santa Rosa. } \\ \text { T. } & \text { = Tauca. }\end{array}$

Algunas categorías geográficas serán abreviadas.

arq. = lugar arqueológico.

c.p. = centro poblado.

\section{REGIÓN YUNGA FLUVIAL}

\section{Fitotopónimos de la región yunga fluvial}

Capulí, El. (*), (1), chacra, (Bol.), capulí. (Del náhuatl capolli). m. Fruto de una planta solanácea silvestre, parecido a un tomatillo, de color naranja, de sabor agridulce, que se consume como fruta (DRAE). NC: Physalis peruviana ${ }^{3}$.

Choloque. (2) paraje y chacra, (Bol., Con.), choloque. m. Árbol pequeño y resistente, de color amarillo claro. Sus frutos redondos y de color negro son utilizados para lavar ropa y también para lavarse el cabello. Nombres comunes: chururo, jabonillo, sulluco. NC: Sapindus saponaria.

Choloque, El. (1), paraje, (Bolog.). Ver Choloque.

Guinda, La. (*), (1), chacra, (Con.), guinda. (Quizá del germ. *wìkšna). m. Árbol de la familia de las Rosáceas, especie de cerezo, del que puede distinguirse por ser las hojas más pequeñas y el fruto más redondo y comúnmente ácido (DRAE). NC: Prunus serotina.

Higuera, La. $\left(^{*}\right),(1)$, chacra, (T.), higuera. (Del lat. ficarı̌a). m. Árbol de la familia de las Moráceas, de tamaño mediano; savia lechosa; hojas grandes con lóbulos desiguales; inflorescencia carnosa con flores diminutas; fruto comestible carnoso y dulce. NC: Ficus carica.

Higuerón. $\left(^{*}\right),(1)$, quebrada, (Pamp.), higuerón. (De higuera). m. Árbol de la familia de las Moráceas, con tronco corpulento, copa espesa, hojas grandes y alternas, fruto de mucho jugo, y madera fuerte, correosa, de color blanco amarillento, muy usada en la América tropical, donde es espontáneo el árbol, para la construcción de embarcaciones (DRAE). NC: Ficus atrox; Ficus spp.

Higuerón, El. (2) puquio y ojonal, (Pamp.). Ver Higuerón.

Hualango. (1), chacra, (Con.), huarango. (Del q. waranqu). m. m. Árbol mediano, copa extendida, espinoso; hojas compuestas finas; flores en cabezuelas amarillas; vainas anchas y encorvadas (DEPUP). NC: Acacia macracantha ${ }^{4}$.

Hualanguito. (1), chacra, (Con.); diminutivo de Hualango. Ver Hualango.

Laurel. (*), (1), quebrada, (Bol.), laurel. (Del prov. laurier). m. Árbol siempre verde, de la familia de las Lauráceas, que crece hasta seis o siete metros de altura, con tronco liso, ramas levantadas, hojas

3 En Pallasca se le llama capulí al aguaimanto.

4 Ni el DRAE ni el DEPUP registran hualango, pero el DEPUP registra la forma huarango. 
coriáceas, persistentes, aromáticas, pecioladas, oblongas, lampiñas, de color verde oscuro, lustrosas por el haz y pálidas por el envés; flores de color blanco verdoso, pequeñas, en grupillos axilares, y fruto en baya ovoidea y negruzca. Las hojas son muy usadas para condimento [...] (DRAE). NC: Laurus nobilis.

Lúcuma. (*), (2) chacras, (Huac. y Pam.), lúcuma. (Del q. lukma). m. Árbol de 6 a 15 m de alto, copa ancha, follaje brilloso; frutos en las ramas jóvenes, redondo o alargado; con punta en la extremidad; piel marrón verde, y muy fina; pulpa amarilla oscura, firme, arenosa y muy dulce; 1 a 2 semillas (DEPUP). NC: Pouteria lucuma.

Lúcumas, Las. (1), chacra, (Cab.). Ver Lúcuma.

Lucumaball $\sim$ Lucumaballe. (3) chacras (Llap. y Pam.), lúcuma y ball(e) ${ }^{5}$. Ver Lúcuma.

Lucumapampa. (1), chacra, (Huand.), lúcuma y pampa ${ }^{6}$. Ver Lúcuma.

Manzanillas, Las. $\left(^{*}\right),(1)$ chacra, (S.R.), manzanilla. f. Hierba de la familia de las Compuestas, con tallos débiles, comúnmente echados, ramosos, de dos a tres decímetros de longitud, hojas abundantes partidas en segmentos lineales, agrupados de tres en tres, y flores olorosas en cabezuelas solitarias con centro amarillo y circunferencia blanca (DRAE). NC: Hymenoxis robusta.

Matibamba. (4), chacras y caserío, (Huac, Con., T.), mati y bamba ${ }^{7}$, mati. (Del q. mati 'calabacita'). m. Árbol ramoso que alcanza unos diez metros de altura cuyo tallo llega hasta $30 \mathrm{~cm}$ de diámetro [...]; hojas simples fasciculadas, pecioladas [...]; inflorescencia, cauliflora; flores solitarias o dispuestas en pares en las ramas, hermafrodita, blanco o blanco amarillentas, fruto pepo o calabaza, de forma esférica u ovoidal, de tamaño variable que va de 8 a $30 \mathrm{~cm}$ de diámetro [...] (DEPUP). NC: Crescentia cujete.

Nogal, El. (*), (1), chacra, (Pam.), nogal. (Del lat. nucālis, semejante o relativo a la nuez). m. Árbol de la familia de las Yuglandáceas, de unos quince metros de altura, con tronco corto y robusto, del cual salen gruesas y vigorosas ramas para formar una copa grande y redondeada, hojas compuestas de hojuelas ovales, puntiagudas, dentadas, gruesas y de olor aromático, flores blanquecinas de sexos separados, y por fruto la nuez. Su madera es dura, homogénea, de color pardo rojizo, veteada [...] (DRAE). NC: Juglans andina.

Olivo. (*), (1), chacra, (Lacab.), olivo. (Del lat. olivum). m. Árbol de la familia de las Oleáceas, con tronco corto, grueso y torcido, copa ancha y ramosa que se eleva hasta cuatro o cinco metros, hojas persistentes coriáceas, opuestas, elípticas, enteras, estrechas, puntiagudas, verdes y lustrosas por el haz y blanquecinas por el envés, flores blancas, pequeñas, en ramitos axilares, y por fruto la aceituna, que es drupa ovoide de dos a cuatro centímetros de eje mayor, según las castas, de sabor algo amargo, color verde amarillento, morado en algunas variedades, y con un hueso grande y muy duro que encierra la semilla. Originario de Oriente, es muy cultivado en España para extraer del fruto el aceite común (DRAE). En Pallasca no se extrae aceite. NC: Podocarpus macrostachyus.

Pitajaya. (*), (1), camino, (Pam.), pitahaya. f. Planta de la familia de las Cactáceas, trepadora y de flores encarnadas o blancas según sus variedades. Algunas dan fruto comestible (DRAE). NC: Selenicereus megalanthus.

Pitajaya, La. (1), chacra, (Huand). Ver Pitajaya.

Pitajayas, Las. (1), paraje, (Bol.).Ver Pitajaya.

5 Ball (o balle en boca de los hispanohablantes) es una palabra culle que significa 'loma'. (Torero 1989), pero también 'hondonada' (Cuba 2005). La forma balle es muchas veces escrita valle (coincidente con la forma castellana valle 'cuenca de un río').

6 Pampa viene del quechua (DQAH).

7 Bamba viene del quechua pampa. Ver nota (6). 
Rocotos, Los. $(*),(1)$, chacra, (Pamp.), rocoto. (Del q. rukutu). m. Planta herbácea de la familia de las Solanáceas que da un fruto grande, de color rojo, verde o amarillo y muy picante (DRAE). NC: Capsicum puvescens.

Sauce. (*), (1), mina, (Pamp.), sauce. (Del lat. salix, -ǐcis). m. Árbol de la familia de las Salicáceas, que crece hasta $20 \mathrm{~m}$ de altura, con tronco grueso, derecho, de muchas ramas y ramillas péndulas. Tiene copa irregular, estrecha y clara, hojas angostas, lanceoladas, de margen poco aserrado, verdes por el haz y blancas y algo pelosas por el envés, flores sin cáliz ni corola, en amentos verdosos, y fruto capsular. Es común en las orillas de los ríos (DRAE). NC: Condaminea corymbosa.

Sausalpampa. (1), paraje, (Con.), sauce + -al y pampa, sausal. Conjunto de sauces. Ver Sauce.

Taya $^{8}$. (2), calle y chacra, (Huand. y Lacab.), tara. (Del q. tara). f. Árbol de hasta $5 \mathrm{~m}$ de altura; de ramas abiertas, espinosas, de corteza gris oscura y las hojas terminales; las flores son en racimos terminales; las vainas son aplanadas [...] (DEPUP). Otros nombres comunes: tara, algarrobo tanino. NC: Caesalpinia spinosa.

Tunas. $(*),(1)$ puquio, (Pam.), tuna. (De or. taíno). f. Especie semejante a la higuera de tuna, silvestre, con más espinas y fruto de pulpa muy encarnada (DRAE). NC: Opuntia ficus-indica.

Tunas, Las. (2), paraje y chacra, (Llap. y Bolg.). Ver Tunas.

Tunaspampa. (1), chac, (Con), tunas y pampa. Ver Tunas.

Uchutranca. (1), chacra, (Con.), uchu y tranca, uchu. (Del q. uchu 'ají, rocoto'). Ver Los Rocotos.

\section{Zootopónimos de la región yunga fluvial}

Pajarito. $\left(^{*}\right),(1)$, laguna, (Pam.); diminutivo de pájaro, pájaro. (Del ant. pássaro). m. Ave, especialmente si es pequeña (DRAE).

\section{REGIÓN QUECHUA}

\section{Fitotopónimos de la región quechua}

Achupalla. (*), (1), paraje, (Lacab.), achupalla. (Del q. achupalla 'una planta', Fam. Bromelia bicolor'). f. Planta silvestre de tallos gruesos, de hojas alternas envainadoras y con espinas en los bordes, flores en espiga, flores moradas y fruto en forma de piña (DRAE). En Pallasca es utilizada como adorno y sus hojas como alimento para cabras y cuyes. NC: Puya pyramidata.

Achupalla, La. (2), falda de cerro y paraje, (Con. y Pam.). Ver Achupalla.

Achupallas, Las. (1), quebrada, (Huand.). Ver Achupalla.

Aliso, El. (*), (2), chacras, (Huac. y Pam.), aliso. (Quizá del gót. *alı̌sa). m. Árbol de la familia de las Betuláceas, de unos diez metros de altura, copa redonda, hojas alternas, trasovadas y algo viscosas, flores blancas en corimbos y frutos comprimidos, pequeños y rojizos (DRAE). NC: Alnus acuminata.

Alisos, Los. (2), chacras, (Bol. y Pam.). Ver El Aliso.

Amaros, Los. (*), (1), lago, (Pam.), amaro. (Del lat. marum). m. Planta de la familia de las Labiadas, de unos siete a ocho decímetros de altura, muy ramosa, con hojas grandes, acorazonadas en la base, recortadas por el margen y cubiertas de un vello blanquizco, y flores en verticilo, blancas con viso morado y de olor nauseabundo. Se usa como tópico para las úlceras (DRAE). NC: Chuquiraga webwrbaueri. 
Chachacoma, La. (1), paraje, (Cab.), chachacoma. (Del q. chachakuma) m. Árbol de la cordillera andina de madera dura, de corteza escamosa, de flores amarillas usado en la confección de herramientas agrícolas y de otros utensilios. Nombres comunes: chachacomo, chachacoma, chachas. N.C. Escalonia resinosa.

Chamana. (1), chacra, (Pam.), chamana. (De or. inc.). f. Es un arbusto, [...] raramente árbol, de $10 \mathrm{~m}$ de altura. Hojas simples, elípticas, [...] que segregan una sustancia resinosa. Las flores van del amarillo al anaranjado rojizo, en racimos cortos. El fruto es una cápsula de $2 \mathrm{~cm}$ de ancho, al madurar es pardo, con tres alas (Wikipedia). NC: Dodonaea bialata.

Changa. (1), chacra, (Huac.), changa. (Probablemente de origen culle) ${ }^{9}$. f. Planta herbácea perenne, aromática, de flores blancas y azulinas, y de hojas verdes; habita en costa, sierra y selva, entre los 500 y 3500 msnm. NC: Minthostachys mollis.

Chilca. $\left.{ }^{*}\right),(2)$, chacra y quebrada, (Pamp.), chilca ${ }^{10}$. (Del q. chhillca, y esta del ai. ch'illka). f. Arbusto de tallos rojizos, hojas verdes y amargas. Es utilizado en la medicina, pero también para teñir el color amarillo o verde (DEPUP). Hay diversas especies. NC: Baccharis lanceolata.

Chilca, La. (1), quebrada, (Huand.). Ver Chilca.

Chilcapuquio. (2), vertiente y ojonal, (Con.), chilca y puquio ${ }^{11}$. Ver Chilca.

Chilcas (2), chacra y pocito de agua, (Pam. y T.). Ver Chilca.

Chilcas, Las. (3), paraje, quebrada y llanura, (Huand.). Ver Chilca.

Culantrillo. (*), (1), chacra, (Con.), culantrillo. Helecho perenne; rizoma escamoso; radículas; peciolos largos, negros, radicales, delgados; hojas bi-tripinnadas, delgadas, verde-amarillentas, lobuladas, en abanico; esporangios en el margen superior de las hojas, color ocre, soros, reniformes (DEPUP). NC: Adiantum capillus-veneris.

Guagor. (1), cerro, (Con.), guagor. (De or. inc.). m. Planta cactácea de color verde, pegada al suelo, cubierta de espinas potentes, de flores de colores que van del amarillo al rojo muy encendido, de frutos. NC: (Es una variedad de Opuntia).

Guagor, La Pampa. (1), pampa, (Cab.). Ver Guagor.

Huarauya. (1), chacra, (Pamp.), huarauya. (De or. inc.). $\mathrm{m}$. Arbusto silvestre, de 3 a $7 \mathrm{~m}$ de alto, de tallos derechos, de hojas más o menos menudas y de flores amarillas acampanadas. Sin la corteza, estos tallos son lisos y utilizados como varas. NC: Tecoma stans.

Huarauyas, Las. (3), quebrada y terrenos, (Cab., Huand. y T.). Ver Huarauya.

Llátures. (2), Paraje donde crecen llátures, (Pall. y Huand.), llatur. (De or. inc.). m. Planta cactácea de gran altura. Habita a una altura de $2800 \mathrm{~m}$ a $3000 \mathrm{msnm}$. NC: No encontrado.

Llaturbara. (1), paraje, (Huac.), llatur y bara ${ }^{12}$. Ver Llátures.

Llaturpata. (2), chacra y desierto, (Pamp.), llatur y pata ${ }^{13}$. Ver Llátures.

Lloques, Los. (1), terreno de cultivo, (Huand.), lloque. m. Arbusto de hasta $4 \mathrm{~m}$ de alto. Flores blanquecinas. Hábitat: Sierra entre 2400 y 3800 msnm. NC: Kageneckia lanceolata.

Marco, El. (2), chacras, (Pam. y Huand.), marco. (Del q. marku) m. Hierba de hojas expandidas de color verde plomizo, utilizado para curaciones bronquiales. NC: Ambrosia peruviana.

9 Changa en quechua es muña.

10 Etimología tomada del Vocabulario de la lengua general de todo el Perú llamada lengua qquichua o del inca. 1989 [1952] de Diego Gonzáles Holguín.

11 Puquio del quechua pukyo (DQAH).

12 Bara en culle significa 'terreno cultivable' (Torero 1989; Cuba 1995).

13 Pata en quechua significa 'parte alta' (DQAH). 
Marcuballe. (2), chacras, (Cab. y Huand.), marco y balle. Ver El Marco.

Marcopampa. (1), chacra, (Pam.), marco y pampa. Ver El Marco.

Molle. $\left(^{*}\right),(1)$, chacra, (Pam.), molle. (Del q. mulli). m. Árbol de mediano tamaño, de la familia de las Anacardiáceas, propio de América Central y Meridional, que tiene hojas fragantes, coriáceas y muy poco dentadas, flores en espigas axilares, más cortas que las hojas, y frutos rojizos. Su corteza y resina se estiman como tonificante nervioso y antiespasmódico (DRAE). En la zona de estudio se usan las hojas en infusión y en baños para curarse de los bronquios. m. Nombres comunes: árbol de la vida, cullash, falsa pimienta, huigan, huiñan, pimienta del Perú, maera, orcco mulli. NC: Schinus molle.

Molle, El. (2), quebrada y loma, (Huand.). Ver Molle.

Molle, Loma del. (1), loma, (Pam.). Ver Molle.

Molle, Pampa del. (1), chacra, (Pam.). Ver Molle.

Mollebamba. (1), chacra, (T.), molle y bamba. Ver Molle.

Mollepampa. (1), chacra, (Con.), molle y pampa. Ver Molle.

Molles, Los. (4), paraje, quebradas y cerro, (S.R. y Pam.). Ver El Molle.

Porocsho. (1), quebrada, (Huand.). (Probablemente del q. puru puru). Ver Purpuro.

Purpuro. (1), chacra, (Pam.), purpuro. (Del q. puru puru 'granadilla ácida'). m. Planta trepadora silvestre. Se encuentra en los valles interandinos hasta $3500 \mathrm{msnm}$ y en la Selva Alta. Nombres comunes: curuba, granadilla silvestre, poro poro, puroqsho, purpuro, purush, shambagol, tacso, tintin, trompos, tumbo (del monte). N.C: Passiflora foetida.

Shambagol. (4), loma, cerro, chacra y caserío, (Bolog., Cab. y Pall.), shambagol. (Probablemente de origen culle). Ver Purpuro.

Shayle, El. (1), chacra (Bol.), shayle. (De or. inc.). m. Planta de $2.5 \mathrm{~m}$ de altura, flores amarillentas o verdes, inflorescencia de $80 \mathrm{~cm}$ de alto, dispuestas en forma de espiga. En su conjunto es una planta pegajosa y de olor no agradable. De uso medicinal. Antiguamente, en la zona, sus hojas han sido utilizadas para reemplazar al tabaco. NC: Nicotiana glutinosa.

Shiraque. (1), quebrada, (Lacab.), shiraque (De or. inc.) m. Árbol de hasta cuatro metros de altura de hojas ovaladas, cuyo hábitat es entre los 2000 y 3000 msnm. Sus hojas son utilizadas como medicina. NC: Lochroma tupayachianum.

Shiracball. (2), parajes, (Lla.), shirac y ball. Ver Shiraque.

Shiraques, Los. (3), quebradas y chacra, (T., Cab. y Pam.). Ver Shiraque.

Shita. (1), chacra, (Lacab.), shita. (De or. inc.) m. Arbusto cuya altura puede alcanzar cinco metros, sus hojas son de color oscuro por el haz y claro por el envés, de aspecto aterciopelado; muy común en los valles de las regiones quechua y suni. NC: No encontrado.

Shita, La. (1), quebrada, (Cab.). Ver Shita.

Shitas, Las. (1), chacra, (Pam.). Ver Shita.

Shitaball. (2), chacra y paraje, (Cab.), shita y ball. Ver Shita.

Shitamaca. (1), chacra, (Lacab.), shita y maca ${ }^{14}$. Ver Shita.

\section{Zootopónimos de la región quechua}

Alljomaca. (1), cerro, (Cab.), alljo y maca, alljo. (Del q. allqo 'perro').

Carnero, Alto. $\left(^{*}\right),(1)$, paraje que involucra algunas chacras, (Cab.), carnero. (Del lat. [agnus] carnarŭus, [cordero] de carne). m. Mamífero rumiante, que alcanza de siete a ocho decímetros de 
altura hasta la cruz, con frente convexa, cuernos huecos, angulosos, arrugados transversalmente y arrollados en espiral, y lana espesa, blanca, negra o rojiza. NC: Ovis orientalis aries.

Challagón. (1), quebrada, (Lacab.), challa y gon ${ }^{15}$, challa. (Del q. challa 'pez pequeño'). m. Pececito de agua dulce, de aproximadamente tres $\mathrm{cm}$., de color gris. Habita en los puquiales. NC: No encontrado.

Huanchaco, Caserón del. (1), chacra, (T.), huanchaco (Del q. wanchaaku). m. Pájaro del tamaño del gorrión, plumas de color plomo, de aspecto jaspeado entre oscuro y claro, con el pecho colorado. NC: Sturnella militaris.

Lacabamba. (2), distrito y quebrada, (Lacab.), laca y bamba, laca. (Del cull. llaga 'venado') ${ }^{16}$. NC: Mazama gouzobuira.

Lacapampa. (1), chacra, (T.), laca y pampa. Ver Lacabamba.

Murciélago. (*), (1), peña, (Lacab.), murciélago. (De murciégalo y este del lat. mur 'ratón' y caecus 'ciego'). m. Quiróptero insectívoro que tiene fuertes caninos y los molares con puntas cónicas. Tiene formado el dedo índice de las extremidades torácicas por solo una o a lo más dos falanges y sin uña. Es nocturno y pasa el día colgado cabeza abajo, por medio de las garras de las extremidades posteriores, en los desvanes o en otros lugares escondidos (DRAE). El lugar es una peña con rendijas profundas donde duermen los murciélagos. NC: Pipistrellus pipistrellus.

Pichungón. (4), terrenos, loma y chacra, (Cab., Huand. y Lacab.), pichun y gon, pichun. (Probablemente del q. pichiw, 'gorrión') ${ }^{17}$. m. Pájaro pequeño, de plumaje plomo con plumas algo anaranjadas que forman un pequeño collar; y plumas negras en la cabeza, a manera de gorro. Nombre común: gorrión andino. N.C.: Zonotrichia capensis.

Tuco, El. (2), peñas, (Huand. y Pam.), tuco. (Del q. tuku 'búho'). m. Ave rapaz nocturna, de aproximadamente $40 \mathrm{~cm}$ de altura, de color negruzco mezclado con plumas blancas y rojizas, con pico corvo, ojos grandes y en la parte anterior de la cabeza, plumas levantadas como orejas. El topónimo refiere a lugares escarpados donde duermen los tucos (búos). NC: Strix omanensis.

Tuco, Peña El. (1), peña, (Pam.). Ver El Tuco.

Tucos, Los. (1), peña, (Bol.). Ver El Tuco.

Tucupina. (1), loma, (Pam.), tuco y -pina. El topónimo refiere a un lugar descampado con muchos arbustos donde suelen habitar muchos tucos (búhos). Ver El Tuco.

Tucupina Chico. (1), peña alta, (Huand.). Ver El Tuco y Tucupina.

Tucupina Grande (1), peña baja, (Huand.). Ver El Tuco y Tucupina.

Ultupuquio. (1), chacra, (Con.), ulto y puquio, ulto. (Del q. ultu 'renacuajo'). m. Larva del sapo, que se diferencia del animal adulto principalmente por tener cola, carecer de patas, respirar por branquias y también por vivir solamente en el agua.

\section{REGIÓN SUNI O JALCA (Y PARTE DE LA REGIÓN PUNA)}

\section{Fitotopónimos de la región suni o jalca (y parte de la región puna)}

Chinchanco. (2), quebrada, (Pam.), chinchanco. (De or. inc.). m. Yerba silvestre cuyo hábitat es de hasta 3000 msnm; es utilizada para el teñido de color amarillo. NC: Lepidium chichacara.

15 Gon viene del culle goñ y significa 'agua' (Martínez Compañón).

16 Laca viene del culle llaga y significa 'venado' (Flores, 2000).

17 Es probable que pichiw al unirse al morfema culle gon haya sufrido una alteración en su límite morfémico y se haya convertido en pichu primero y luego, al fusionarse con la siguiente palabra haya buscado una armonía consonántica nasal: pichun, es decir, pichungón. 
Chocho. $\left(^{*}\right),(1)$, chacra, (Pam.), chocho. (Del mozár. šóš, y este del lat. salsus, salado, por prepararse así habitualmente). m. altramuz ( II fruto) (DRAE). Planta leguminosa de frutos comestibles, de la familia de las Fabáceas. Debido a su fuerte amargor, debe pasar por un proceso de cocimiento y de varios días de enjuague en agua corriente, antes de su consumo. Nombres comunes: taurhui / tarwi, chuchus muti. El topónimo refiere a una enorme chacra donde produce buenos frutos de chochos. NC: Lupinus mutabilis. Ver Tauribara.

Chugallmaca. (1), loma, (Cab.), chúgall y maca, chúgall ${ }^{18}$. (Probablemente de origen culle). m. Planta pequeña, pegada al suelo; sus raíces contienen una sustancia jabonosa. Es utilizada como purgante y también para lavar la ropa. NC: No encontrado.

Chugán Chugañ, El. (1), chacra, (Cab.), chugañ ${ }^{19}$. (Probablemente de origen culle.). m. Arbusto de ramificación espinosa, de consistencia dura, de frutos pequeños de color rojo, de consistencia de la manzana verde. El topónimo se refiere a una chacra rodeada de chuganes. Mayormente esta planta es utilizada para impedir entradas a las chacras con sementera en maduración. NC: Hesperomeles cuneata.

Chuganes. (1), camino, (Lacab.). Ver Chugán.

Chugur. (1), chacra (Lacab.), chugur ${ }^{20}$. (Probablemente de origen culle). m. Planta silvestre cuyo tamaño, forma y disposición de hojas es semejante a la del tarwi o chocho, con diferencia en el tono del color y en el tamaño de las vainas. Mientras que el tarwi es verde intenso con vainas grandes, el chugur es verde claro pálido, más cercano al celeste, con vainas y frutos muy pequeños en relación al primero. Nombre común: tarwi silvestre. NC: Lupinus ballianus.

Chugur, El. (2), chacra y mina, (Huand. y Lacab.). Ver Chugur.

Chugures, Los. (2), chacras, (Con. y Huand.). Ver Chugur.

Chugurball. (1), cerro, (Lacab.), chugur y ball. Ver Chugur.

Cortadera. $\left({ }^{*}\right),(2)$, paraje y ojonal,(Pamp.y Con.), cortadera. (Del cast. 'cortar').f. Planta Ciperácea de hojas alternas, largas, angostas y aplanadas, cuyos bordes cortan como una navaja. Tiene flores rojizas y baya amarilla. Se cría en lugares pantanosos y se usa el tallo para tejer cuerdas y sombreros (DRAE). En Pallasca, la cortadera crece en los pantanos y lagunas y es utilizada básicamente para techar las casas en los caseríos y estancias. NC: Escleria melaleuca, Cortaderia spp.

Cortaderas, Las. (3), chacra y manantiales, (Pam. y T.). Ver Cortadera.

Huáchaper, Alto. (1), arq., cerro, (Pam.), huáchaper. (De or. inc.) Tipo de papa silvestre, de sabor amargo. En la localidad, los lugareños extraen estos tubérculos de los alrededores de la zona arqueológica que se ubica en un cerro, algo alejado de la ciudad y preparan de esta papa el chuño. NC: No encontrado.

Pacra. (2), parajes, (Con., Pam.), pacra. (Del q. paqra). f. Planta silvestre de corola amplia de color verde claro y estambres y pistilo amarillos, que crece en la Sierra (Cajamarca, Ancash) sobre las rocas calcáreas, sobre los $4000 \mathrm{msnm}$. Posee los atributos de planta «cálida». En la zona es utilizada para curar el reumatismo y para alimentar a las reses con el fin de obtener una mejor reproducción. Otros nombres comunes: pacra-pacra, pagra. NC: Laccopetalum giganteum.

Pacracucho. (1), cerro, (Pamp.), pacra y cucho ${ }^{21}$, pacra. Ver Pacra.

Pacras. (1), cerro, (Pamp.). Ver Pacra.

18 Muchas palabras culle terminan en lateral palatal $[\lambda]$ o en nasal palatal [ñ ] (Cuba 2004).

19 Ver nota 18.

20 Chugur es una palabra culli (Flores, 2000). El chugur es una planta de la que se supone es la variedad no domesticada del tawri o chocho. 
Pagua. (1), quebrada, (Pam.), pagua. (De or. inc.). f. Planta pequeña de flor amarilla y de hojas menudas, con flores en capítulo amarillo. Se le utiliza para teñir de color amarillo. Otro nombre: sullcash. NC: Coreopsis senaria.

Panizara. (1), cerro, (Huan.), panizara. (De or. inc.). f. Arbusto silvestre de hojas pequeñas, de un verde más intenso en el haz que en el envés y flores que crece en la Sierra Norte, encima de 2500 m. La infusión de la planta tiene efecto digestivo, carminativo, antiespasmódico (Ver DEPUP). Otros nombres comunes: panicera, orégano de los incas. NC: Satureja pulchella.

Quinual, El. (1), laguna (Cab), quinual. (Probablemente de origen quechua). m. Árbol de la familia Rosácea, silvestre y cultivado en la Sierra; de madera dura y de hojas menudas que se disponen alrededor de peciolos cortos. (Ver el DEPUP). En la localidad su madera es usada como leña y para elaborar herramientas de labranza. Nombres comunes: quiñuar, qqeuña, quinua. NC: Polylepis incana.

Quinuacocha Quinualcocha. (2), paraje y laguna, (Con.), quinual y cocha ${ }^{22}$, quinual. Ver El Quinual.

Quinuales. (1), quebrada, (Con.). Ver El Quinual.

Quinuales, Laguna. (1), laguna, (Con.). Ver El Quinual.

Tauribara. (*), (1), caserío, (Huand.), tauri y bara, tauri. (Del q. tarwi) ${ }^{23}$. Planta leguminosa oriunda del Perú, que crece hasta un metro de altura y produce unos granos en vaina comestibles llamados chochos. Ver Chocho.

Totora. $\left(^{*}\right),(1)$, chacra, (Lacab.), totora. (Del q. tutura, 'totora'). f. Planta perenne, común en esteros y pantanos, cuyo tallo erguido mide entre uno y tres metros, según las especies. Tiene uso en la construcción de techos y paredes para cobertizos y ranchos (DRAE). El topónimo está asignado a un espacio cultivable, al pie de una laguna cubierta de totoras. NC: Typha angustifolia.

Totora, Laguna. (3), lagunas, (Huand., Cab. y Pam.). Ver Totora.

Totoras, Las. (3), cerro y quebradas, (Con.). Ver Totora.

Upaiguero, El. (1), quebrada, (Huand.), upaiguero. (De or. inc.). m. Planta silvestre de aproximadamente $70 \mathrm{~cm}$, cuyas hojas menudas forman copos alrededor del tallo. Es utilizada para teñir de color marrón (oque). NC: No encontrado.

\section{Zootopónimos de la región suni o jalca (y parte de la región puna)}

Atojorco. (1), chac, (Cab.), atoj y orco ${ }^{24}$, atoj. (Del q. atoq 'zorro'). zorro. m. Mamífero cánido de aproximadamente un metro de longitud, incluida la cola, de hocico alargado y orejas paradas, pelaje de color pardo rojizo, cola densa y punta blanca. Es de costumbres nocturnas y caza con gran astucia. El topónimo corresponde a un lugar de difícil acceso, aunque de buena tierra para el cultivo. Los pobladores mayores que conocían el lugar tan testimonio de la existencia de zorros en dicho lugar. NC: Vulpes vulpes.

Atojchaca. (1), chacra, (Con.), atoj y chaca ${ }^{25}$, atoj. Ver Atojorco.

Cárgache. (2), cueva y chacra ,(Con.), cárgache. (De or. inc.). m. Ave del tamaño de un pollo mediano; cuerpo cubierto de plumas grises; alas y patas amarillas; vive en las cumbres de los cerros o en cuevas, a más de 3500 msnm. NC: No encontrado.

22 Cocha del quechua qucha 'laguna' (Íbidem).

23 El DRAE registra tarhui. En la zona de estudio la palabra ha pasado por una metátesis: [tár.wi]>[táw.ri] (grafémicamente: tar.hui > tahu.ri > tauri).

24 Orqo del quechua urqu 'cerro' (DQAH).

25 Chaca del quechua chaka 'puente' (Íbidem). 
Cernícalo. (*), (1), chacra, (Pam.), cernícalo. (Del lat. cernicŭlum, criba). m. Ave de rapiña, [...], de unos cuatro decímetros de largo, con cabeza abultada, pico y uñas negros y fuertes, y plumaje rojizo más oscuro por la espalda que por el pecho y manchado de negro (DRAE). NC: Falco tinnunculus.

Cernícalo, Cueva del. (1), cueva, (Lacab.). Ver Cernícalo.

Cóndor, Piedra El. (*), (2), peñas, (Lacab. y Pam.), cóndor. (Del q. kúntur). m. Ave rapaz del orden de las Catartiformes, de poco más de un metro de longitud y de tres de envergadura, con la cabeza y el cuello desnudos, y en aquella carúnculas en forma de cresta y barbas; plumaje fuerte de color negro azulado, collar blanco, y blancas también la espalda y la parte superior de las alas; cola pequeña y pies negros. Habita en los Andes y es la mayor de las aves que vuelan (DRAE). NC: Vultur gryphus.

Condorcocha. (3), lago, mina y cerro, (Con.), cóndor y cocha. Ver Piedra El Cóndor.

Condorera. (1), quebrada, (Con.), cóndor +-era. Risco, escarpado donde solían vivir los cóndores. Ver Piedra El Cóndor.

Condormuchca Condormúchica, Toma. (2), toma y quebrada, (Con.), cóndor y muchca ${ }^{26}$, cóndor. Ver Piedra El Cóndor.

Condorsenga . (1), cerro, (Lacab.), cóndor y senga ${ }^{27}$, cóndor. Ver Piedra El Cóndor.

Guanaco. $\left(^{*}\right),(1)$, paraje, (T.), guanaco. (Del q. wanaku). m. Mamífero rumiante de unos trece decímetros de altura hasta la cruz [...]. Tiene cabeza pequeña con orejas largas y puntiagudas, ojos negros y brillantes, boca con el labio superior hendido, cuello largo, erguido, [...], cuerpo de abundante pelo largo y lustroso, de color generalmente pardo oscuro, [...]; cola corta [...], patas delgadas y largas, con pies de dos dedos bien separados y con fuertes uñas [...]. Es animal salvaje que habita en los Andes meridionales (DRAE). El topónimo alude a un lugar donde solían pastar los guanacos. Actualmente no existen guanacos. NC: Lama guanicoe.

Huaicho. (2), chacra y peña, (Huac. y Lacab.), huaicho. m. Ave palmípeda, que vive cerca a las lagunas de la cordillera. Tiene plumas oscuras, pecho blanco y patas coloradas. NC: No encontrado.

Huaicho, Cuello de. (2), quebrada y riachuelo, (Con.). Ver Huaicho.

Huaicho, Peña El. (1), peña, (Con.). Ver Huaicho.

León, El. (*), (2), quebrada y hoyo, (Lla.), león ${ }^{28}$. En Pallasca no ha existido ni existe el león sino el puma ${ }^{29}$.

Llama, Alto. (*), (1), arq., (Pam.), llama. (Del q. llama). f. Mamífero rumiante, variedad doméstica del guanaco, del cual solo se diferencia en ser algo menor, pues tiene un metro de altura hasta la cruz, y aproximadamente igual longitud. Es propio de América Meridional (DRAE). NC: Lama glama.

Llama, El Alto. (1), cerro, (Pam.).Ver Alto Llama.

26 Muchca múchica del quechua muchka 'mortero' (Íbidem).

27 Senga del quechua sinqa 'nariz'. (Íbidem).

28 León. (Del lat. leo, -ōnis). m. Gran mamífero carnívoro de la familia de los Félidos, de pelaje entre amarillo y rojo. Tiene la cabeza grande, los dientes y las uñas muy fuertes y la cola larga y terminada en un fleco de cerdas. El macho se distingue por una larga melena (DRAE). NC: Panthera leo.

29 Poma. (*) (Del quechua puma.) m. Felino americano de la familia Felidae; de unos $180 \mathrm{~cm}$ de longitud, de color rojizo o leonado uniforme, que vive en las serranías y llanuras (DRAE). Es el segundo mayor felino en el mundo después del jaguar, y el cuarto más grande del mundo. También conocido como león de montaña (Wikipedia). NC: Puma concolor. 
Mishu Chico. (1), laguna, (Con.), mishu ${ }^{30}$. (Del q. mishu 'gato'.) m. Mamífero carnívoro de la familia de los Félidos, de aproximadamente ochenta centímetros de largo desde la cabeza hasta el arranque de la cola. Tiene cabeza redonda, lengua muy áspera, patas cortas y pelaje espeso, suave, gris, pardo, rojizo o negro. El lugar posee una laguna rodeada de arbustos que se ubica al pie de otra laguna más grande: Mishu Grande, que también está rodeada de arbustos. NC: Felis silvestris catus.

Mishu Grande. (1), laguna, (Con.). Ver Mishu Chico.

Shingo, Piedra. (1) chacra con piedra en forma de shingo, (Pam.), shingo. (Del q. shinku 'gallinazo'). f. Ave rapaz diurna que se alimenta de carroña, de $60 \mathrm{~cm}$ de longitud, cabeza y cuello sin plumas, de color gris oscuro, cola corta. Vive en la cordillera. El topónimo es motivado, pues el lugar refiere a una chacra que tiene una piedra en forma de shingo o gallinazo con las alas extendidas. NC: Stephanoaetus coronatus.

Tógope. (2), cerros, (Cab. y Huand), tógope. (De origen onomatopéyico). m. Ave del tamaño de una paloma de castilla, de cuerpo gris cenizo; se le conoce con ese nombre por su canto. NC: No encontrado.

Vizcacha. $\left({ }^{*}\right),(1)$, cerro, (Con.), vizcacha. (Del q. wisk'acha, 'roedor andino'). f. Roedor parecido a la liebre, de aproximadamente $80 \mathrm{~cm}$, con cola larga como la del gato, de color plomizo, que vive en los lugares escarpados, en pedregales, en la cordillera. NC: Lagotis criniger.

Vizcachas, Las. (4), peña, chacra, cerros, (Bolg., Cab., Con. y Huand.). Ver Vizcacha.

Vizcachas, Puente Las. (1,) puente, (Pam.). Ver Vizcacha.

\section{REGIÓN RUPARUPA O SELVA ALTA}

\section{Fitotoponimia de la región ruparupa o Selva Alta}

Café, El. $\left(^{*}\right),(1)$, chacra, (Con.), café. (Del it. caffe, este del turco kahve, y este del ár. clás. qahwah). $\mathrm{m}$. Semilla del cafeto, como de un centímetro de largo, de color amarillento verdoso, convexa por una parte y, por la otra, plana y con un surco longitudinal (DRAE). NC: Coffea arábica.

Caña brava. $\left(^{*}\right),(1)$, chacra, (Con.), caña brava. f. Gramínea silvestre muy dura, con cuyos tallos se hacen tabiques y se emplean en los tejados para sostener las tejas. NC: Gynerium sagitattum.

Cedro. $(*),(2)$, quebrada y puquio, (Con.), cedro. (Del lat. cedrus). m. Árbol de la familia de las Abietáceas, que alcanza unos $40 \mathrm{~m}$ de altura, con tronco grueso y derecho, ramas horizontales, hojas persistentes casi punzantes, flores rojas al principio y después amarillas, y cuyo fruto es la cédride (DRAE). NC: Caryocar barbinerve.

Cedro, El. (1), quebrada, (Con.). Ver Cedro.

Cedro, Río de. (1), río, (Con.) Ver Cedro.

Cedroball $\sim$ Cedroballe. (2) quebrada y barrio, (Pam.), cedro y ball(e), cedro. Ver Cedro. También sobre ball balle ver nota 5 .

Ñuñabamba. (1), chacra, (Con.), ñuña y bamba, ñuña. (Probablemente de origen culle). f. Planta muy variable en el porte, desde baja y postrada hasta trepadora, por la gran variedad de cultivares, adaptados a las condiciones ambientales diferentes; hojas superiores alternas, tres folíolos; inflorescencia en racimo axilar, con flores simétricas, de colores generalmente blancos; legumbre falcada, recta o curva, aplanada, ápice encorvado o recto; semillas blancas o de colores (DEPUP). NC: Phaseolus vulgaris.

30 En este caso mishu equivale a un gato montés. 
Palo Santo. (*), (1), chacra (Con.) m. Árbol de la misma familia que el guayaco, cuya madera, muy dura, se emplea en ebanistería y tornería. Tiene también aplicaciones medicinales (DRAE). Muchas variedades. NC: Trichanthera gigantea, Bursera graveolens, Dalbergia spp.

\section{Aporte DE OTRAS INVESTIGACIONES VINCULADAS CON EL TEMA}

El trabajo Aportaciones a la fitotoponimia de la provincia de Ciudad Real, de García-Villaraco, Pardo y Morales (2006) es el que más afinidad tiene con nuestro trabajo, pues se trata de una primera recopilación de datos de índole botánica, etnobotánica y etnoecológica en la provincia de Segovia. Dichos fitotopónimos «informan sobre paisajes y especies vegetales que pueden haber desaparecido, aclarando aspectos de usos del territorio en cuestión o la forma de ver los paisajes que tienen o tuvieron sus gentes».

Aparte de tomar en cuenta solo fitotopónimos y no zootopónimos, este trabajo se diferencia esencialmente del nuestro en la metodolología, ya que los datos son recopilados a partir de los mapas militares que corresponden a dicha provincia y a partir de lo cual los autores asumen que dichos fitotopónimos «informan sobre paisajes y especies vegetales que pueden haber desaparecido [resaltado nuestro], aclarando aspectos de usos del territorio en cuestión o la forma de ver los paisajes que tienen o tuvieron sus gentes». En nuestro caso, la recopilación de datos ha sido in situ, a partir de informantes, y lo que hemos verificado es la vigencia, la supervivencia de términos de lenguas de pueblos desaparecidos.

Un aporte importante que se rescata del trabajo de García-Villaraco et al. es que Los fitotopónimos se ubicaron mediante coordenadas UTM de precisión de $1 \mathrm{~km}$ de los nombres obtenidos. En nuestro trabajo aún falta hacer esta tarea.

El los demás aspectos, la descripción del conjunto de fitotopónimos se desarrolla casi siguiendo las mis pautas que nosotros hemos adoptado, aunque variado en detalles muy particulares, pues, de alguna manera relacionamos la toponimia con la lexicografía.

Otro de los trabajos relacionados con el tema es Nombres en el paisaje: la toponimia, Fuente de conocimiento y aprecio del territorio de Pascual Riesco Chueca (2001). El autor echa mano de muchos trabajos centrados en la fitoponimia y zootoponimia (aunque precisamente no use esta terminología) como el estudio de García Fernández. Riesco no solo se centra en los elementos del paisaje sino que muestra una visión más profunda sobre lo que significa la toponimia en relación con el paisaje. Se refiere a la densidad toponímica como dato esencial en los usos y la historia del lugar; también sostiene que los topónimos son «expresión de cultura y biogeografía, tradición oral y literatura»; así como distingue ‘dos dimensiones' casi antagónicas del nombre de lugar: como el de carácter hereditario versus aquel de posible imposición, por razones simbólicas o de poder.

\section{CONCLUSIONES}

Del análisis de los topónimos estudiados se puede destacar tres aspectos principales.

1. En cuanto a su naturaleza: En la muestra de topónimos hay más referencias de vegetales que de animales. De 136 topónimos; 100 (73,53\%) contienen nombres de plantas y 36 (26,47\%), de animales.

2. En cuanto a su distribución: Los topónimos son un vivo testimonios de entidades fitonímicas y zoonímicas que se desarrollan dentro de una determinada región, aunque en varios casos puedan extenderse a más de una. De la muestra extraída de topónimos se ha verificado que el grueso de plantas y animales aparece en la región quechua: 44 (32,35\%) nombres de plantas y 13 (9,56\%), de animales. Le sigue la región suni o jalca: 26 (19,12\%) nombres de plantas y 26 (19,12\%), de animales. Después la yunga, con 29 (21,32\%) nombres de plantas y el 1 (0,74\%), de animal. Por último, la región ruparupa, con solo 8 (5,88\%) nombres de plantas. 
3. En cuanto a su etimología:Los nombres de plantas y animales, en su gran mayoría son de etimología quechua: 65 (47,79\%); de origen castellano, 35 (25,74\%); de origen culle, 11 (8,09\%) y de origen incierto, 25 (18,38\%). Dentro del primer grupo (quechua) hay; 17 nombres de plantas: achupalla, chachacoma, chilca, hualango, hualanguito, marco, mati, molle, pacra, porocsho, purpuro, quinual, rocoto, tauri, taya, totora, uchu; 13 de animales: alljo, atoj, challa, cóndor, guanaco, huanchaco, llama, mishu, pichu, shingo, tuco, ultu. Dentro del segundo grupo (castellano) hay; 20 nombres de plantas: capulí, guida, higuera, higuerón, manzanilla, palo santo, laurel, nogal, olivo, sauce, sausal, aliso, amaro, culantrillo, chocho, cortadera, café, caña brava, cedro; 5 de animales: carnero, cernícalo, león, murciélago, pajarito. Dentro del tercer grupo (culle) hay 6 nombres de plantas: changa, chugall, chugañ, chugur, ñuña y shambagol; 1 de animal: laca. Dentro del cuarto grupo (or. inc.) hay 14 nombres de plantas: choloque, chamana, guagor, huarauya, llatur, lloque, shayle, shirac, shita, chinchanco, huáchaper, pagua, panizara, upaiguero (varios de estos quizá de origen culle); 3 nombres de animales: cárgache, huaicho y tógope. La presencia de las palabras quechua y culles, junto a las castellanas revela la identidad de los pobladores pallasquinos. La conservación de estas formas originarias puede explicarse como una forma de resistencia al avasallamiento de la cultura hispana.

4. Los nombres de plantas que se repiten con más frecuencia en los topónimos son: molle, chilca, tunas, shita, quinual, shirac y llatur. Los nombres de animales que aparecen con mayor frecuencia en este corpus son: tuco, vizcacha y cóndor.

5. Finalmente, este estudio constituye un aporte para la biología, en la medida que da algunos indicadores de las características de las plantas y animales y del medio en que viven.

\section{BibliografíA}

ADELAAR, Willem F. H.

1987 «Search for the Culle Language». Ponencia presentada al $1 .^{\text {er }}$ Congreso Nacional de Investigaciones Lingüístico-Filológicas, Lima, noviembre.

AFABLE, P.O. y M.S. BEELER

1996 «Place Names». En: I. Goddard (ed.) Languages, Vol. 17: 90-95, of Handbook of North American Indians, ed. W.C. Sturtevant. Washington, D.C., Smithsonian Institution.

AGAPITO, Teodoro e Isabel SUNG

1998 Fitomedicina. 1100 plantas medicinales. Lima: Editorial Isabel IRL.

AGUSTINOS

1918 Relación de idolatrías en Huamachuco por los primeros agustinos. Lima, Colección de libros y documentos referente a la Historia del Perú, Ed. Urteaga y Romero, T. XI.

ANDRADE, Luis

1999 «Topónimos de una lengua andina extinta en un listado de 1943». Lexis 23 (2): 401-425.

BRACK EGG, Antonio

1999 Diccionario Enciclopédico de Plantas Útiles del Perú. Lima: PNUD y Cusco: Centro de Estudios Regionales Andinos «Bartolomé de las Casas».

BÜTTNER, Thomas y Dionisio CONDORI

1984 Diccionario Aymara-Castellano / Arunakan Liwru Aymara-Kastillanu. Puno: Proyecto Experimental de Educación Bilingüe-Puno.

CARVAJAL LAZO, Herman.

1998 Ovalle y la toponimia indígena del Limarí. Sotaqui: Editorial Caburga.

CUBA, María del Carmen.

2004 «Algunos rasgos gramaticales y semánticos del castellano de Huandoval». En: Actas del Simposio «Centenario de José Jiménez Borja». Descripción y enseñanza del español, pp. 139-157. Lima: Cátedra UNESCO para la lectura. 
1995 «Toponimia de Pallasca» En: Cuadernos de la Asociación Cabanista. Año 2, № 13.

2005 «Morfología toponímica de la provincia de Pallasca». En: Escritura y Pensamiento. Revista de la Unidad de Investigaciones, Facultad de Letras y Ciencias Humanas, UNMSM, Año VIII, N 16, pp. 113147.

FLORES REYNA, Manuel

2000 «Recopilación léxica preliminar de la lengua culle». Tipshe [Facultad de Humanidades, Universidad Federico Villarreal] 1, 1, noviembre, pp. 173-197.

GARCÍA-VILLARACO, Antonio, Manuel PARDO DE SANTAYANA y Antonio Ramón MORALES

2011 «Aportaciones a la fitotoponimia de la provincia de Ciudad Real». Revista del Folklore 357: 4-23. Madrid: Departamento de Biología, Facultad de Ciencias, Universidad Autónoma de Madrid, Real Jardín Botánico, CSIC. [En línea]. < http://digital.csic.es/bitstream/10261/66938/1/363FitonimiaC RRevFolk.pdf > [consultado el 14/02/2014].

GONZÁLEZ HOLGUÍN, Diego

1989 Vocabulario de la lengua general de todo el Perú llamada lengua qquichua o del inca. Lima: Universidad Nacional Mayor de San Marcos. (Edición facsimilar de la versión de 1952).

INSTITUTO GEOGRÁFICO NACIONAL DEL PERÚ

s/f Carta Nacional (escala 1: 100.000), hojas: 17-g (Santiago de Chuco), 17-h (Pallasca) y 18-g (Santa Rosa), en el sistema UTM.

MARTÍNEZ COMPAÑóN, Baltasar Jaime

1978 [1790] Trujillo del Perú en el siglo XVIII. Vol. 2. Madrid: Cultura Hispánica.

MENÉNDEZ PIDAL, Ramón

1952 Toponimia prerrománica hispana. Madrid: Ed. Gredos.

PARKER, Gary y Amancio CHÁVEZ

1976 Diccionario Quechua Ancash-Huailas. Lima, Ministerio de Educación-Instituto de Estudios Peruanos.

PINCHEMEL, Philippe y Genevieve PINCHEMEL

1979 Réflexions sur l'histoire de la géographie: histoires de la géographie, histoire des géographies. París: Comité des travaux historiques et scientifiques.

PULGAR VIDAL, Javier

2007 Las 8 regiones naturales del Perú. Lima: Universidad Alas Peruanas.

SILVA SANTISTEBAN, Fernando

1982 «La lengua culle de Cajamarca y Huamachuco». Cantuta 9. Lima: Universidad Nacional de Educación Enrique Guzmán y Valle, La Cantuta.

TORERO C., Alfredo

1989 «Áreas toponímicas e idiomas de la sierra norte peruana (Un trabajo de recuperación lingüística)». Revista andina 7(1): 217-249. Cusco, Centro de Estudios Regionales Andinos «Bartolomé de las Casas».

TORT, J.

2003 «A propòsit de la relació entre toponímia i geografia: el principi de 'significativitat territorial'». Butlletí Interior de la Societat d'Onomàstica 94-95: 675-688. 